\title{
Une épidémie se propage et personne ne s'en inquiète
}

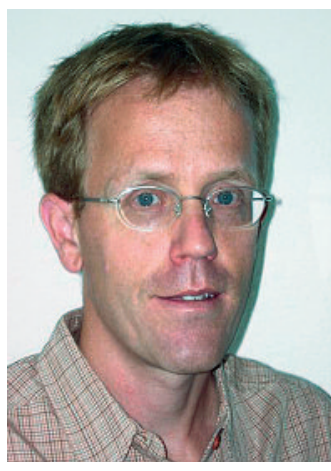

Philip Bruggmann
Correspondance:

Dr Philip Bruggmann

Directeur Somatik ARUD Zürich

Poliklinik Zokl1

Sihlhallenstrasse 30

CH-8026 Zürich

P.Bruggmann@arud-zh.ch
Près de quatre décès sur dix constatés l'année dernière chez les patients traités à la méthadone dans la région de Zurich étaient dus à une insuffisance hépatocellulaire. A chaque fois, une infection, non traitée, par le virus de l'hépatite $C$ était en jeu. Les trois quarts des dépendants aux opiacés ont contracté une hépatite $\mathrm{C}$ alors que seule la moitié des patients traités à la méthadone dans le canton de Zurich a été informé convenablement des risques de l'hépatite $\mathrm{C}$ et moins de 10\% a suivi une thérapie. Pourquoi cette épidémie chez les patients toxicodépendants n'intéresse-t-elle pas les médecins? Est-ce en raison du manque de lobby de la part des patients, ou de nos lacunes dans la manière de se comporter face aux maladies addictives ou encore des structures de soins médicaux insuffisantes à l'égard des consommateurs de stupéfiants?

Au début de l'épidémie du VIH au sein de la population toxicomane, plusieurs mesures ont été mises en œuvre avec réussite, conduisant à une sensible réduction du nombre de nouveaux cas (programme de substitution à la méthadone, remplacement des seringues, salles d'injections). Ces mesures se sont révélées être insuffisantes pour empêcher ou réduire la propagation de l'hépatite $C$ puisque l'incidence n'a pratiquement pas diminué. A l'inverse de nos pays voisins, l'Aide suisse contre le SIDA ne se préoccupe guère de cette épidémie chez les consommateurs de drogues. En raison de la similitude des voies de contamination de la maladie, un rapprochement serait judicieux. En fin de compte, les médecins sont en excellente compagnie.

Ces dernières années ont démontré qu'il est tout à fait possible de soigner avec succès des toxicomanes contre l'hépatite $\mathrm{C}$, indépendamment de leur consommation actuelle. Ce que nous pouvons confirmer grâce à nos propres expériences. Souvent, les traitements à prescrire ne peuvent pas l'être dans le cadre thérapeutique traditionnel, quelques-uns des toxicodépendants n'étant pas en mesure de prendre place dans une salle d'attente de médecin. Les comorbidités psychiques sont fréquentes et requièrent une offre thérapeutique modulable au cas par cas et compacte qui, dans la mesure du possible, concentre une large palette de soins médicaux sur un même site, à l'instar des policliniques de l'ARUD (Arbeitsgemeinschaft für risikoarmen Umgang mit Drogen) à Zurich ou dans certains cabinets médicaux spécialisés. Ces offres sont souvent inexistantes dans beaucoup de régions suisses. Tous les spécialistes impliqués (gastro-entérologues, spécialistes de maladies addictives, psychiatres et médecins de famille) sont invités à s'attaquer à ce problème, soit par le biais de services de consultation consacrés à l'hépatite $C$, soit dans des réseaux spéciaux autorisant une étroite collaboration.

Mandatée par l'OFSP, Infodrog a initié cette année une campagne nationale de sensibilisation pour permettre aux médecins, patients, collaborateurs d'institution de prévention et de dépendances d'approfondir leurs connaissances au sujet de l'hépatite $C$ (www. hepch.ch). Cette campagne propose une base informative et poursuit l'objectif de réduire l'épidémie d'hépatite $\mathrm{C}$ au sein du groupe marginal des toxicomanes pour, entre autres, apporter des éléments de réponse aux conséquences considérable qui s'annoncent sur les plans économique et sanitaire. Sur ce point, beaucoup de pays occidentaux sont logés à la même enseigne que la Suisse. Ils prennent progressivement la mesure des coûts potentiels inhérents au nombre croissant de cirrhoses du foie chez les toxicodépendants, et ce notamment par le biais de confrères qui soignent aujourd'hui déjà des patients toxicomanes atteints d'hépatite $C$. C'est ainsi qu'est né un réseau international de spécialistes à l'initiative de l'ARUD Zurich qui, études multicentriques à l'appui, s'est fixé pour objectif d'énoncer des fondements scientifiques supplémentaires utiles à l'élaboration de notices d'instruction internationales, de promouvoir l'échange du savoir et des expériences entre les pays et ainsi d'améliorer la prévention en matière d'hépatite $C$ pour augmenter les taux de dépistage et de thérapie chez les toxicomanes.

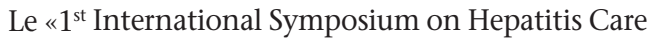
in Substance Users», organisé à Zurich les 24 et 25 septembre par l'ARUD représentera un forum idéal pour approfondir son savoir et échanger ses expériences avec des confrères du monde entier. Outre des conférences sur tous les sujets importants de ce domaine, tenues par des orateurs de renommée internationale, pas moins de 200 médecins issus des cinq continents sont attendus pour débattre (www.arud.ch/ symposium).

Chères collègues et chers confrères, ces maladies méritent que nous leur portions un regard plus attentif. La médecine de l'addiction et les cas d'hépatite C chez les toxicodépendants ouvrent sur des domaines multidisciplinaires et passionnants, recélant encore vbeaucoup a déjà été fait et beaucoup reste encore à faire.

Philip Bruggmann 